\title{
Study of bubbling fluidization dynamics via Digital Image Analysis Technique
}

\author{
A. Busciglio, G. Micale, L. Rizzuti \& G. Vella \\ Dipartimento di Ingegneria Chimica, dei Processi e dei Materiali, \\ Università degli Studi di Palermo, Italy
}

\begin{abstract}
In the area of chemical engineering, fluidization is a widely employed technology for a number of industrially important processes. A sound understanding of the mechanisms governing the complex flow phenomena involved in a fluidized bed still remains an open technical and scientific issue.

The principal difficulty in analysing fluidization quality and bubble dynamic is concerned with the possibility of measuring or predicting the physical and geometrical properties of gas bubbles chaotically rising in a solid granular medium.

In recent years the use of Computational Fluid Dynamics (CFD) has been significantly increasing to simulate multi-phase flows. It is invariably emphasized that a necessary step towards the development of reliable fully predictive CFD models is an extensive experimental validation of the simulation results.

On the above basis, the present work focuses on the statistical analysis of the behaviour of a 2-D fluidized bed operating under bubbling and slugging conditions. Experimental data are collected by the means of a purposely developed Digital Image Analysis Technique (DIAT). DIAT is capable of measuring several bubbles properties simultaneously. Moreover it could be used without any modification for computational data analysis, thus providing the answer to the need for close comparison between experimental and computational data. In fact, in many cases, direct experimental validation of CFD simulation is difficult and cumbersome, since different level of detail is available in experimental and computational investigations. In this work, the development of an image analysis technique available for both experimental and computational data does allow direct and effective quantitative comparison.
\end{abstract}

Keywords: multiphase flow, CFD, image analysis. 


\section{Introduction}

Many of the characteristic features of gas-fluidized beds, like the excellent solid mixing, heat and mass transfer properties, can be related to the presence of bubbles and are dominated by their behaviour. A deeper knowledge of the fluidized bed hydrodynamics and on how such hydrodynamics are affected by the operative conditions, especially geometry changes and plant scale-up, would provide the base for the development of a fully predictive model describing the gas solid flow in a fluidized bed.

The gas bubbles rising up through a typical fluidized bed ensure that the particles are circulated throughout the bed so that properties and process condition could be considered uniform, but at the same time could eventually allow gas bypass, depending on operative conditions. They have a considerable importance in the fluidized solid-gas systems because they govern hydrodynamics and efficiency of the operation for which the bed is used.

The recent development of mathematical modelling of particulate solids behaviour together with the increased computing power enables researchers to simulate the behaviour of fluidized powders and to link fundamental particle properties directly to the powder behaviour and predict the interaction between particles and gaseous or liquid fluids. In this regard, Computational Fluid Dynamics (CFD) modelling provides a fundamental tool to support engineering design and research in multiphase systems. Many Authors recognise that computational modelling in multi-phase systems has the potential to increase process efficiency and reduce the number of scale-up steps in the design of reliable commercial plants.

It is invariably emphasized that a necessary step towards the development of reliable fully predictive CFD models is an extensive experimental validation of the simulation results. In the first instance, the experimental validation will come to assess the CFD model chosen to simulate the investigated regimes.

On the above basis, the present work focuses on the simulation of a 2-D fluidized bed operating under bubbling and slugging conditions carried out with the use of the commercial CFD code Ansys CFX-10.0. Computational results are post-processed by a Digital Image Analysis Technique, [1], and compared with experimental data.

\section{Literature review}

Eulerian-Eulerian continuum modelling of multiphase flows, with the fluid and solid phases treated as interpenetrating continuum phases, is the most commonly used approach for fluidised bed simulations, [2]. The general idea in formulating the multi-fluid model is to treat each phase as an interpenetrating continuum, and therefore to construct integral balances of continuity, momentum and energy for both phases, with appropriate boundary conditions and jump conditions for phase interfaces. Since the resultant continuum approximation for the solid phase has no equation of state and lacks variables such as viscosity and normal stress [2], certain averaging techniques and assumptions are required to obtain a complete 
momentum balance for the solids phase. In the last decades many investigators tried to develop a theory of particle collision based on the kinetic theory approach by [3]. The solid-phase momentum equation contains an additional term to account for momentum exchange due to particle-particle collisions. The absence of the stress term of the particle phase in the particulate momentum equation has led to different models adopting different closure methods, including the kinetic theory model [4-6]. The application of the kinetic theory to model the motion of a dense collection of nearly elastic spherical particles is based on an analogy to the kinetic theory of dense gases. A granular temperature, $\Theta$, is defined to represent the specific kinetic energy of the velocity fluctuations or the translational fluctuation energy resulting from the particle velocity fluctuations. In granular flow, particle velocity fluctuations about the mean are assumed to result in collisions between particles being swept along together by the mean flow. The granular particle temperature equation can be expressed in terms of production of fluctuations by shear, dissipation by kinetic and collisional heat flow, dissipation due to inelastic collisions, production due to fluid turbulence or due to collisions with molecules, and dissipation due to interaction with the fluid [5].Numerous studies have shown the capability of the kinetic theory approach for modelling bubbling fluidized beds (e.g. $[2,4,6,7$, 8]).

Once developed appropriate models for the explicit formulation of solid phase stress tensor, the inter-phase momentum transfer between gas and solid phases is still needed to mathematically close the problem. The momentum exchange (represented by a drag force) is one of the dominant forces in the gas- and solidphase momentum balances. Numerous correlations for calculating the momentum exchange coefficient of gas-solid systems have been reported in the literature, including those of Syamlal and O'Brien [9], Gidaspow [5], and Wen and $\mathrm{Yu}[10]$.

\section{Experimental set-up}

The fluid-bed reactor purposely designed and built for the present investigation is made of Perspex ${ }^{\circledR}$ with dimensions equal to 800 (height) x 180 (width) x 15 (depth) $\mathrm{mm}$. The whole experimental setup is shown in figure 1(a).

The reactor is therefore almost two-dimensional, thus allowing visual observations of bubble dynamics within the bed. A plastic porous distributor, whose thickness is equal to $10 \mathrm{~mm}$, is placed at the bottom of the particle bed. Below the distributor a wind box allows to equalize the gas flow.

Glass ballotini in the size range $212-250 \mu \mathrm{m}$ were used for the experimental runs with density equal to $2500 \mathrm{~kg} / \mathrm{m}^{3}$. The particles were filled up to a bed height of $360 \mathrm{~mm}$, i.e. twice the bed width. The value of $\mathrm{u}_{\mathrm{mf}}$ was experimentally determined and found equal to $5.24 \mathrm{~cm} / \mathrm{s}$. Also the value of gas voidage has been experimentally determined and found equal to 0.385 . In these conditions the fluidized bed had a typical Geldart Group B system behaviour for both particulates. Air was used as fluidizing gas, at a velocity equal to 3.4 times the $\mathrm{u}_{\mathrm{mf}}$. 


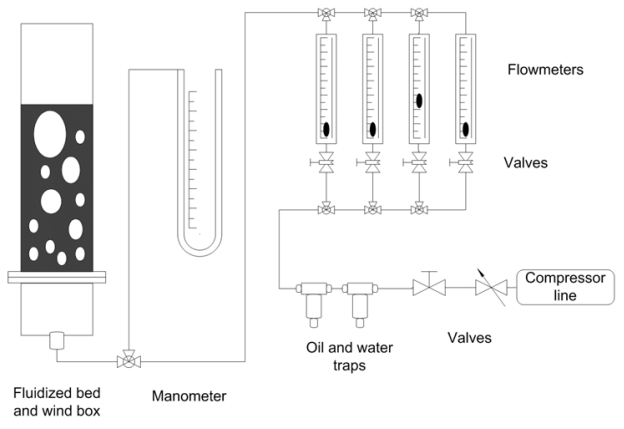

Figure 1: Experimental set-up.

The bubble-related flow structures were visualized with the aid of a continuous back-lighting device obtained by placing six fluorescent lamps at the back side of the bed, at approximately $10 \mathrm{~cm}$, and recorded by a commercial digital camcorder (Sony, model DCRTRV530E PAL), placed opposite to the bed at a distance of $270 \mathrm{~cm}$. The digital visual acquisition system allowed to collect images of the bed at a frequency of $25 \mathrm{~Hz}$. Each experimental acquisition provides at least 500 frames, equal to 20 seconds of real time experiment. Preliminarily, the measurement device was accurately calibrated by means of a purposely generated set of still images that included horizontal and vertical scales, with rectangular, circular and ellipsoid objects. The image processing routine was developed on Matlab 7.0 (The MathWorks inc.), using the Image Processing Toolbox. Thanks to the flexibility of the Matlab environment almost all steps in image processing, data acquiring and elaboration could be easily automated. The reader is referred to Busciglio et al., [1], for full details on the experimental set-up adopted and measurement techniques.

\section{Computational models and methods}

In this study the Eulerian-Eulerian Multi-phase Flow Model (MFM) coupled with the Granular Kinetic Theory (GKT) has been adopted to study the behaviour of gas-solid fluidized beds. This choice is a standard option of the presently adopted CFD code Ansys CFX-10.

Continuity and momentum balance equations are thus solved for each phase using a classical Eulerian-Eulerian description. The standard GKT model is adopted for estimating rheological properties of fluidized solid phase and standard Wen and $\mathrm{Yu},[10]$, model is adopted for estimate the momentum exchange between phases at the phase boundaries.

Because of the similarities between particle-particle interactions and molecular interactions in a gas, the concepts from gas kinetic theory can be used to develop a model for the solids stress tensor. Complete details on the derivations and applications to dense phase flow can be found in Gidaspow [5]. 
The particulate phase is modelled as a population of identical, smooth and inelastic spheres. Particle-particle interactions are described as a binary instantaneous collisions, resembling those between gas molecules.

As far as the numerical aspects are concerned, CFD simulations were performed in a 2D fashion choosing a computational grid consisting of $5 \mathrm{~mm}$ square cells, with $36(\mathrm{X}) \times 288(\mathrm{Y})$ cells. Thus the width of the computational domain exactly coincides with that of the real lab-scale bed previously detailed, conversely the height of the computational domain in fact is bigger than the real bed (i.e. $800 \mathrm{~mm}$ ) in order to apply a fully developed flow condition at the top of the freeboard. The lateral walls were modelled using the standard no-slip boundary condition. The upper section of the simulated geometry, or freeboard, was considered to be occupied by gas only. A simple pressure boundary condition was imposed at the top of freeboard (i.e. fully developed flow condition). A Dirichlet boundary condition was employed at the bottom of the bed to specify a uniform vertical gas inlet velocity throughout the distributor. Symmetry planes were imposed on the front and rear faces of the simulated bed.

The initial conditions specify only the distribution of solid volume fraction within the bed of solids which was set equal to 0.65 .

Typical running CPU times for CFX10 simulations were equal to about 100 hrs for 10 seconds of real time simulated with a fixed time step interval $\Delta \mathrm{t}=10^{-4} \mathrm{~s}$ on a Dell Dimension 8300 Personal Computer.

The simulated case is identical to that experimentally investigated.

\section{Post-processing procedure via Digital Image Analysis Technique}

The quantitative data on experimental and simulated bubble dynamics have been obtained by the means of an original Digital Image Analysis Technique, [1].

This is based on bed images, suitably obtained with backlighting in order to enhancing the contrast between the emulsion phase and the bubble phase. Bubbles in the bed are detected because they create transparent areas in the flat cross-sectional plane of the bed, through which light, emitted at the back of the bed can pass through and reach the camera.

The problem of phase separation enhancing in experiments does not exists in simulation. As a matter of fact, it is possible to obtain, by the mean of code postprocessor, full coloured concentration maps. Images ready for Image Processing can be obtained by superimposing, as output of post-processor, black and white images, in which areas having solid concentration less than the conventional value of 0.15 are drawn as white areas and other areas are drawn as black ones.

By means of the DIAT, geometric properties of all bubbles found in the image are measured. The above mentioned Digital Images Analysis Technique (DIAT) can be easily used for both experimental images and computational volume fraction maps, without any adjustment. The data obtained can be used to rebuild some important statistical information about the bubble dynamics, such as: 
i) Statistical probability density of bubble equivalent diameters in the whole bed and as function of bubble distance from the distributor;

ii) Average bubble number as function of the distance from the distributor;

The rising velocity distribution can be found by comparing bubble centroids positions in subsequent frames. Within the present work a Lagrangian Velocimetry Technique (LVT) has been adopted, [1], able to locally track each bubble in his chaotic rising motion along the bed.

The data processed through the use of the LVT allow to rebuild fundamental information on bubble dynamics, such as:

i) variation of bubble rise velocity with bubble diameter, as obtained by smoothing processing of raw data by means of a bisquare weight function ;

ii) Statistical probability density of bubbles rise angles;

The direct rigorous comparison between experimental and computational data on bubble dynamics so obtained will allow coherent validation of computer simulation of bubbling fluidized beds.

\section{Results and discussions}

In Figure 2.a and figure 2.b the experimental and computational distributions of bubble size distribution are reported. A characteristic positive skewed distributions are generally observed. This kind of distribution is in accordance with the relevant literature, [11].

It is worth noting that a single skewed distribution, such as Gamma distribution or Log-normal distributions are not able to correctly follow data trend. Theoretical analyses, [12], have shown that Gamma distribution can be obtained if a coalescence-driven bubble enlargement is considered.

On the other hand, complex nucleation and splitting phenomena generally give rise to significant amount of bubbles with smaller diameters at any distance from distributor, thus leading to a first (almost constant) peak at smaller diameter, and a moving peak typical of coalescence-enlarging bubbles.

This considerations lead us to consider, as fitting curves, the linear combination of Gamma distributions shown in Equations 1, that shows excellent fitting.

$$
p d f\left(d_{b}\right)=\varphi \frac{1}{\gamma_{2}{ }^{\gamma_{1}} \Gamma\left(\gamma_{1}\right)} d_{b}^{\gamma_{1}-1} e^{-\frac{d_{b}}{\gamma_{2}}}+(1-\varphi) \frac{1}{\gamma_{4}^{\gamma_{1}} \Gamma\left(\gamma_{3}\right)} d_{b}^{\gamma_{3}-1} e^{-\frac{d_{b}}{\gamma_{4}}}
$$

where $\Gamma$ is the integral Gamma function.

$$
\Gamma(m)=\int_{0}^{\infty} e^{t} t^{m-1} \mathrm{dt}
$$

The numerical predictions appear to qualitatively estimate the complex bimodal trend of bubbles size distribution, even if the quantitative agreement may still improve. 
(a)
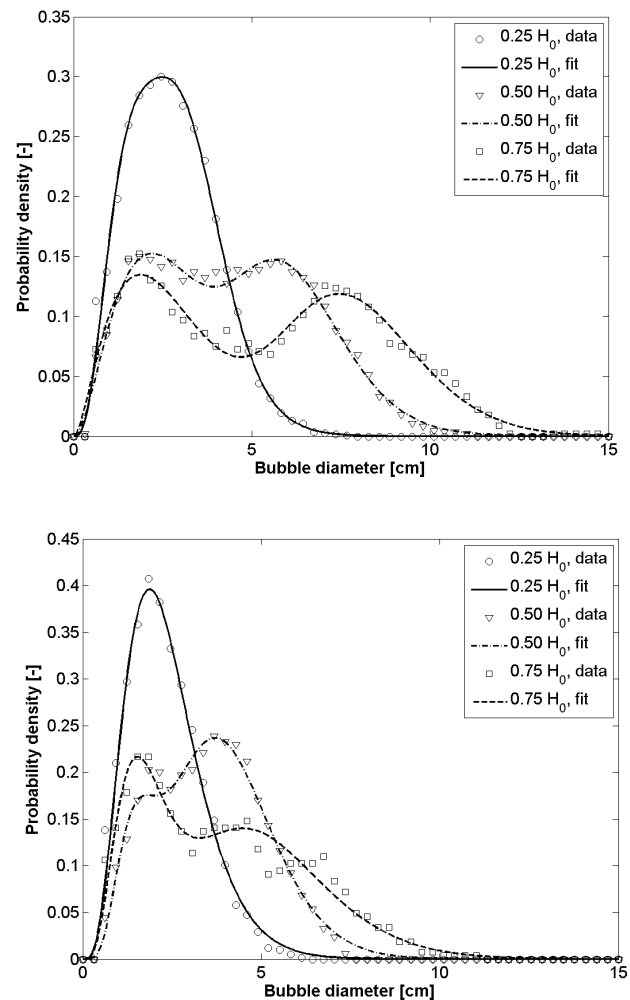

(b)

Figure 2: Experimental (a) and computational (b) probability density distributions of bubble diameter at different distances from distributor and relevant curve fitting.

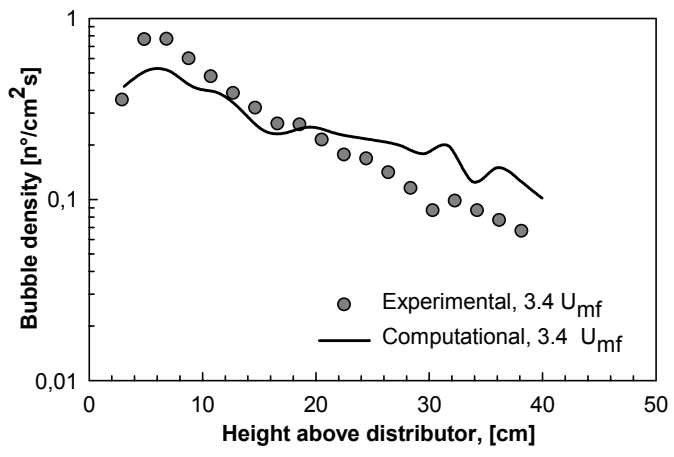

Figure 3: Bubble density along bed height. 
Figure 3 reports the experimental and computational bubble density profiles along the bed height. It must be pointed out that a linear decay of bubble density in a logarithmic chart would characterize a constant bubble coalescence rate, while a constant value of bubble density would be that typical of fully developed slug flows.

The experimental data trend is characterized by a clear linear decay, related to a net constant decay rate of bubble density along bed height. Such trend appear to be sufficiently predicted by the code, even if somewhat lower decay rate is predicted.

As far as the experimental raw rise velocity data are concerned, a significant scattering has been observed. This is not surprising at all given the chaotic behaviour typical of bubbling fluidized beds. Therefore a choice was made to report in Figure 4 only the smoothed version of the rise velocity data. It can be noted that the numerical predictions are in satisfactory agreement with experimental data, particularly for the general trend. Moreover a comparison with the power law proposed by Davidson [13], $u_{b}=\varphi\left(g_{b}\right)^{0.5}$ shows that the correlation acceptably predicts the trend of bubble rise velocity increase with increasing bubble diameter, although some general level of overestimation is found at all ranges.

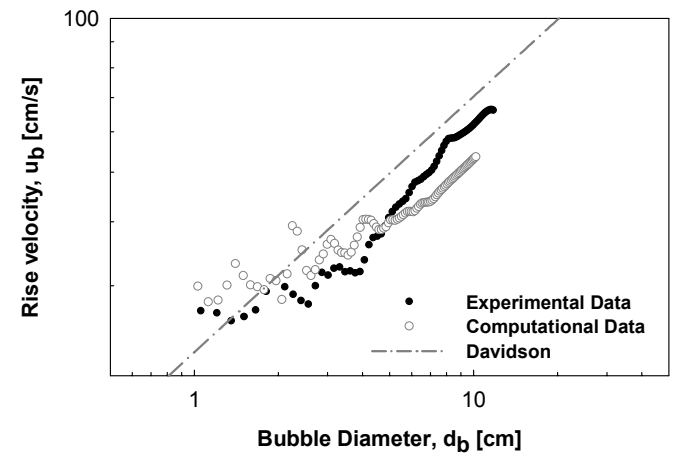

Figure 4: Smoothed distribution of bubble rise velocities.

In Figure 5 the probability distributions of rising angle for both experimental and computational data are reported. It must be noted that the rising angles always follow an almost symmetrical distribution, with the average value near the vertical rise angle, as physically expected.

Given the random lateral motion of bubbles a sort of Gaussian distribution may be expected to fit the data. However careful observation of the shape of the distribution, with respect to the relevant Gaussian fit curve, highlights a positive excess kurtosis. This can be principally due to the near wall bubbles, with limited random lateral motion. It is thus expected that fluidized beds with larger width could present rise angle distributions more similar to Gaussian distributions. The comparison of the computational distribution with the experimental one shows 
an acceptable agreement along most of the distribution interval, while significant underestimation of the probability density is found around the mean value of the distribution.

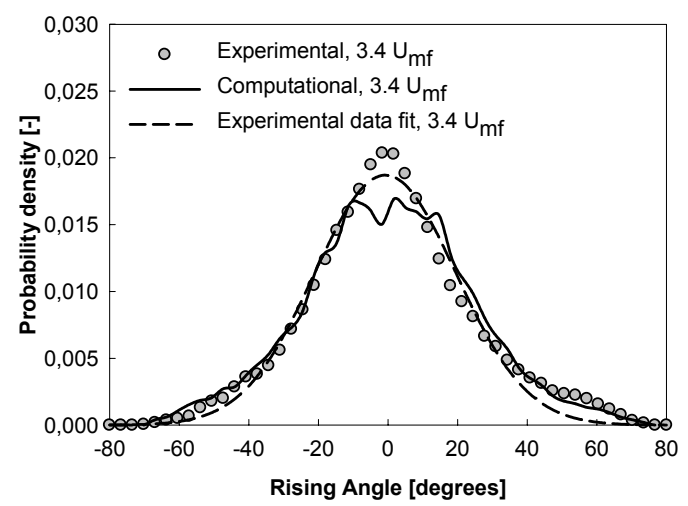

Figure 5: Probability density of bubble rise angle.

\section{Conclusions}

The direct comparison between experimental and computational data on bubble dynamics obtained by means of a Digital Image Analysis Technique allowed the validation of the predictive capability of the Ansys CFX 10.0 CFD code.

In particular the validation of CFD predictions with experimental data focused on the analysis of selected bubbles characteristics, namely the bubble diameter probability density function, bubble density, bubble rise velocity and bubble rise angle.

The statistical significance of the results, due to the substantial quantity of processed data, allowed to identify interesting features of fluidized bed dynamics, such as the bimodality of the local bubble size distribution or the positive excess kurtosis of rise angle distributions. In this regard the code capability in predicting such complex behaviours has been tested with encouraging results.

The present work draws the conclusion that the actual state of the art in mathematical modelling and computer simulation of bubbling fluidized beds allows the qualitative prediction of several phenomena, but major quantitative improvements are still an open task.

\section{References}

[1] Busciglio A., Vella G., Micale G., Rizzuti L., Analysis of the bubbling behaviour of 2-D gas fluidized beds. Part I: Digital Image Analysis Technique, Chemical Engineering Journal, doi:10.1016/j.cej.2007.11.015, 2007. 
[2] Pain C.C., Mansoorzadeh S., de Oliveira C.R.E., A study of bubbling and slugging fluidised bed $s$ using the two-fluid granular temperature model, International Journal of Multiphase Flow, 27, pp. 527-551, 2001.

[3] Chapman S., Cowling T.G., The mathematical theory of non-uniform gases, Cambridge University Press, Cambridge, UK, 3rd edition 1970.

[4] Sinclair J.L., Jackson R., Gas-particle flow in a vertical pipe with particleparticle interactions, AIChEJ, 35, pp. 1473-1486 1989.

[5] Gidaspow D., Multiphase flow and fluidization, Academic Press, San Diego, 1994.

[6] Hrenya C. M., Sinclair J. L., Effects of particle-phase turbulence in gassolid flows, AIChE J., 43, pp. 853-869, 1997.

[7] Ding J., Gidaspow D., A bubbling fluidization model using kinetic theory of granular flow, AIChE Journal, 36, pp. 523-538 1990.

[8] Gelderbloom S.J., Gidaspow D., Lyczkowski R.W., CFD simulations of bubbling/collapsing fluidized beds for three Geldart groups, AIChE J., 49, pp. 844-858, 2003.

[9] Syamlal M., O’Brien T. J., Computer simulation of bubbles in a fluidized bed, AIChE Symposium Series, 85, pp. 22-31, 1989.

[10] Wen C.Y., Yu Y.H., Mechanics of fluidization, Chemical Engineering Progress Symposium Series, 62, pp. 100-111, 1966.

[11] Rowe P.N., Yacono C., The distribution of bubble size in gas fluidised beds, Trans. Instn. Chem. Engrs., 53, pp. 59-60 1975.

[12] Argyriou D.T., List H.L., Shinnar R., Bubble growth by coalescence in gas fluidized beds, AIChE J., 17, pp. 122, 1971.

[13] Davidson J.F., Harrison D., Fluidised Particles, Cambridge University Press, 1963. 